\title{
Associação da Perda Auditiva Induzida pelo Ruído com o Tempo Acumulado de Trabalho entre Motoristas e Cobradores
}

\author{
Noise-Induced Hearing Loss and Its Association with \\ Cumulative Working Time among Urban Bus Workers
}

\author{
Ricardo Cordeiro' \\ Euclydes C. Lima-Filho' \\ Lilian C. R. Nascimento ${ }^{3}$
}

\begin{abstract}
CORDEIRO, R; LIMA-FILHO, E. C. E NASCIMENTO, L. C. R. Noise-Induced Hearing Loss and Its Association with Cumulative Working Time among Urban Bus Workers. Cad. Saúde Públ., Rio de Faneiro, 10 (2): 210-221, Apr/fun, 1994.

Through regression analysis the relationship between noise-induced hearing loss and variables "total cumulative working time", "arterial blood pressure" and "age" was examined. The study was carried out among 278 bus drivers and ticket takers, users of an occupational health center in Campinas, São Paulo State (Brazil).
\end{abstract}

The main results were a positive association between hearing loss and cumulative working time, as well as an interaction between this variable and the bus workers' age.

Key words: Epidemiology; Regression Analysis; Noise; Hearing Loss; Worker's Health

\section{INTRODUÇÃO}

Tradicionalmente, os estudos epidemiológicos que envolvem populações de trabalhadores têm-se ocupado em pesquisar associações plausíveis, do ponto de vista fisiopatológico, entre patologias e supostos fatores de risco (Cordeiro, 1991).

Neste sentido, é notável a produção de trabalhos que estudam associações entre a perda auditiva de origem neurossensorial (particularmente a perda auditiva induzida pelo ruído) e fatores tais como exposição ocupacional a níveis elevados de pressão sonora (Astete \& Kitamura, 1980); diabetes mellitus (Friedman et al., 1975; Harner,

\footnotetext{
${ }^{1}$ Departamento de Saúde Pública da Faculdade de Medicina de Botucatu. Campus de Botucatu, s/n, Botucatu, SP, 18618-000, Brasil.

${ }^{2}$ Departamento de Estatística do Instituto de Matemática, Estatística e Ciências da Computação da Universidade Estadual de Campinas. Caixa Postal 6065, Campinas, SP, 13081-970, Brasil.

3 Programa de Saúde do Trabalhador de Campinas, Secretaria Municipal de Saúde. Av. Anchieta 200, $11^{\circ}$ andar, Campinas, SP, 13015-904, Brasil.
}

1981; Hodgson et al., 1987); fumo (Siegelaub et al., 1974; Barone et al., 1987); viscosidade sangüínea (Browning et al., 1986); hipertensão arterial sistêmica (Talbott et al., 1985); níveis plasmáticos de colesterol livre, gamaglobulina e cortisol (Rai et al., 1981); excreção urinária de catecolaminas (Cesana et al., 1982); atividade plaquetária da monaminoxidase (Cesana et al., 1983); pigmentação da íris (Carter, 1980; Attias \& Pratt, 1985) etc.

Em trabalho publicado em 1987, Laurell \& Noriega, ao estudarem a distribuição de doenças entre operários de uma siderúrgica, notaram que estas se relacionavam não particularizadamente a um ou outro risco especíico, mas sim a uma composição deles. Além disso, os autores estratificam os trabalhadores de acordo com o tempo de trabalho na siderúrgica, notando que os grupos com maior tempo de trabalho apresentavam uma prevalência significativamente maior de várias patologias. Este método parece bastante interessante como uma abordagem preliminar na investigação das relações que se estabelecem entre a doença e o trabalho.

Avançando por este caminho, procurou-se 
neste estudo distanciar-se da tradicional busca de associações entre doenças profissionais e fatores de risco mais ou menos específicos, optando-se por uma abordagem um pouco mais genérica.

O objetivo desta pesquisa foi estudar uma eventual associação entre a perda auditiva induzida pelo ruído e o tempo acumulado de trabalho em meio a condutores urbanos (motoristas e cobradores do transporte coletivo urbano).

\section{MATERIAL E MÉTODO}

A população estudada foi constituída por motoristas e cobradores de veículos coletivos urbanos da cidade de Campinas, São Paulo, usuários do Ambulatório de Saúde Ocupacional do Programa de Saúde do Trabalhador (PST) de Campinas.

O PST, através de seu ambulatório, presta atendimento em saúde ocupacional por intermédio de uma equipe multidisciplinar e alguns recursos técnicos especializados. A demanda deste ambulatórios é, em sua maior parte (cerca de 70\%), encaminhada pelos sindicatos que fazem parte da comissão gerenciadora do PST. O restante é demanda referida pela rede pública ambulatorial de atenção à saúde e demanda espontânea.

Motoristas e cobradores são considerados pelo PST expostos profissionalmente a altos níveis de ruído, e por isso o ambulatório tem por rotina solicitar exame audiométrico a todo profissional desta categoria quando de sua primeira avaliação clínica.

Entre fevereiro de1987 — data da inauguração do PST - e junho de 1992 - quando os dados referentes a esta pesquisa foram coletados - , o ambulatório do PST realizou 292 exames que foram encaminhados ao serviço.

A média de atendimentos/mês da demanda ambulatorial para esta categoria foi de 5,25 casos novos durante o ano de 1992 . O número aproximado de motoristas e cobradores em atividade na área urbana de Campinas neste período foi de 2800 trabalhadores.

Os resultados dos exames audiométricos foram classificados em oito categorias diferentes, segundo a escala de Merluzzi et al. (1979).

Os autores propõem (Merluzzi et al., 1979) que os indivíduos com limiar auditivo bilate- ral igual ou inferior a $25 \mathrm{~dB}$ HTL (ISSO 1990/ 75 e ANSI 1969) para qualquer freqüência testada sejam classificados como "grupo 0" (audiometria normal).

Nos grupos 1, 2, 3, 4 e 5 são agrupados todos os casos de déficit auditivo provocados por ruídos, sendo indicados segundo a gravidade, respectivamente, como hipoacusia de primeiro, segundo, terceiro, quarto e quinto grau.

Nos grupos 6 e 7 se situam, independentemente da gravidade, os traçados que indicam, respectivamente, um déficit auditivo de etiopatogenia mista (ruído mais outra causa) ou de origem diversa do ruído.

A Tabela 1 apresenta a classificação acima referida:

TABELA 1. Status Audiométrico de 292 Condutore, Segundo a Classificação de Merluzzi et al.

\begin{tabular}{lrrc}
\hline \hline Classificação & $\#$ & $\%$ & $\%$ \\
\hline & & & Acumulada \\
Grau 0 & 139 & 47,6 & 47,6 \\
Grau 1 & 66 & 22,6 & 70,2 \\
Grau 2 & 37 & 12,7 & 82,4 \\
Grau 3 & 23 & 7,9 & 90,8 \\
Grau 4 & 1 & 0,3 & 91,1 \\
Grau 5 & 5 & 1,7 & 92,8 \\
Grau 6 & 7 & 2,4 & 95,2 \\
Grau 7 & 14 & 4,8 & 100,0 \\
\hline Total & 292 & 100,0 & \\
\hline \hline
\end{tabular}

Na classificação de Merluzzi, o grau 7 é reservado para as perdas auditivas não causadas por ruído (tais como as perdas auditivas de condução, as provocadas por medicação ototóxica, as de origem infecciosa etc.). Os 14 trabalhadores classificados como grau 7 foram afastados do estudo uma vez que se desejou estudar focalizadamente apenas os portadores de perdas auditivas originadas da exposição ao ruído.

A população estudada constituiu-se portanto de 278 condutores da cidade de Campinas, usuários de um serviço de saúde ocupacional, insentos de qualquer alteração autiditiva nãoneurossensorial.

Observaram-se as seguintes variáveis, quando da primeira avaliação clínica realizada no PST: 
a) Idade (anos)

b) Tempo total acumulado de trabalho como condutor (meses)

c) Pressão diastólica $(\mathrm{mmHg})$

d) Limiar auditivo no ouvido direito a $4000 \mathrm{~Hz}$ $(\mathrm{dB})$

A informação sobre o tempo de trabalho como condutor era obtida rotineiramente, através de entrevista com o trabalhador, quando de sua primeira avaliação clínica feita no PST, informação esta posteriormente ratificada através da análise dos registros feitos em sua carteira profissional.

Quanto à pressão diastólica, esta era obtida através da análise das anotações em prontuário do trabalhador, relativas à primeira consulta.

Quanto à última variável, optou-se pelo limiar auditivo a $4000 \mathrm{~Hz}$ por ser esta a freqüência mais sensível aos efeitos da exposição crônica a ruído intenso, onde costumam ocorrer as primeiras alterações do limiar auditivo na perda auditiva induzida pelo ruído (Hungria, 1987) - fenômeno conhecido como "gota acústica”. A opção pela medida no ouvido direito se deveu à hipótese de ser este ouvido mais precocemente afetado entre os condutores. Neste contexto, portanto, o limiar auditivo no ouvido direito a $4000 \mathrm{~Hz}$ é um sinalizador da perda auditiva induzida pelo ruído. Os valores foram obtidos através da realização de audiometria tonal por via aérea e via óssea em ambos os ouvidos com autiômetro portátil Dicton $C A T$ 741 , calibrado segundo a norma "ISO R.389-
1964" nas freqüências de 500, 1000, 2000, 3000, 4000, 6000 e $8000 \mathrm{~Hz}$, em cabine acústica.

$\mathrm{Na}$ população estudada as variáveis "tempo total de trabalho acumulado como condutor" e "limiar auditivo" se associam ambas com a variável de confundimento "idade". Tais associações são ilustradas pelas Figuras 1 e 2 .

Para a verificação da existência de associação entre o limiar auditivo e o tempo acumulado de trabalho, a opção metodológica feita foi utilizar a técnica de regressão linear múltipla — tendo como variáveis independentes a idade (Id), a pressão diastólica (Pd) e o tempo total de trabalho acumulado enquanto condutor urbano (T), e como variável dependente o limiar auditivo no ouvido direito a $4000 \mathrm{~Hz}$ (LD4) que possibilitou a identificação particularizada da influência da idade, pressão diastólica e tempo de trabalho sobre a variável LD4.

A variável pressão arterial diastólica foi incluída no modelo de regressão devido às referências no sentido de associá-la à perda auditiva induzida pelo ruído (Parvizpoor, 1976; Jonsson \& Hansson, 1977; Manninen \& Aro, 1979; Fouriaud et al., 1984; Talbott et al., 1985; Wu et al., 1987; Kristensen, 1989).

A variável sexo, que classicamente é uma variável confundidora na maioria dos estudos de associação, não fez parte do modelo, uma vez que a população estudada é toda ela do sexo masculino.

Deste modo, o modelo utilizado foi:

$$
L D 4_{i}=\alpha+\beta_{1} I d_{i}+\beta_{2} P d_{i}+\beta_{3} \ln \left(T_{i}\right)+\beta_{4} I d_{i} \ln \left(T_{i}\right)+\varepsilon_{i}(1)
$$

onde a variável LD4 é explicada através de uma combinação linear das variáveis $\mathrm{Id}, \mathrm{Pd}$ e do logaritmo natural da variável $\mathrm{T}$, bem como da interação entre este e a variável Id (representado na equação (1) pelo termo de interação " $\beta_{4}$ $\operatorname{Id}_{\mathrm{i}} \operatorname{In}\left(\mathbf{T}_{\mathrm{i}}\right)$ ".

Aplicou-se uma transformação logarítmica à variável T como recurso para reduzir sua dispersão e aproximar sua distribuição da distribuição normal.

Conforme observa-se na Figura 4, a distribuição dos tempos de trabalho como condutor urbano (T) da população estudada é bastante assimétrica, com mediana (48 meses) bem a esquerda da média (67,19 meses). Além disso, esta distribuição apresenta grande dispersão $\left(\right.$ variância $=3866,19$ meses $\left.^{2}\right)$. Após a transformação logarítmica desta variável, isto é, após converter cada valor de $\mathrm{T}$ em $\ln (\mathrm{T})$, obtém-se uma redução drástica da dispersão dos dados (variância $=1,92[\ln (\text { mês })]^{2}$ ) e uma aproximação boa da simetria, com mediana $(3,87$ $\ln$ [mês]) bastante próxima da média $(3,59$ $\ln [$ mês] $)$. 
FIGURA 1. Tempo Total de Trabalho Acumulado X Idade na População Estudada

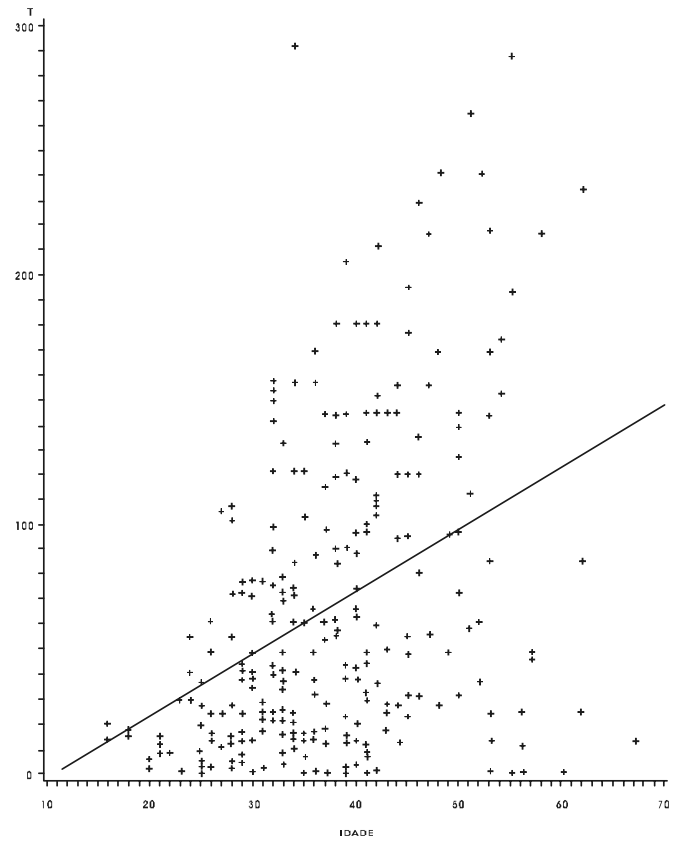

(T) - Tempo Total de Trabalho Acumulado em Meses

Idade - Idade em anos

Obs.: Cada "+" pode representar mais de uma observação

FIGURA 2. Limiar Auditivo X Idade na População Estudada

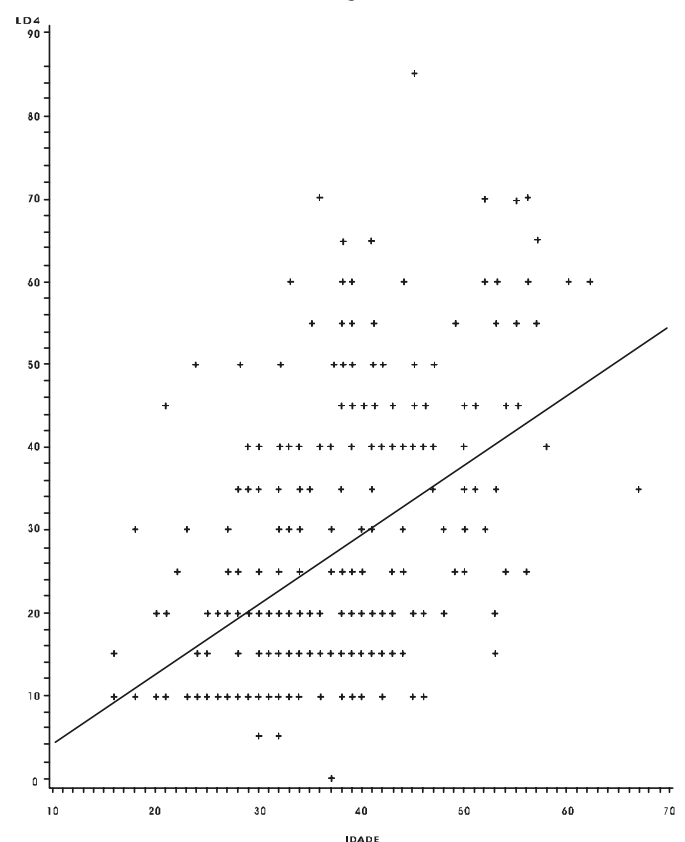

Limiar auditivo medido no ouvido direito $-4000 \mathrm{~Hz}$

(LD4) Limiar Auditivo em dB

Idade - em anos

Obs.: Cada "+" pode representar mais de uma observação 
Através da técnica de regressão linear múltipla ajustou-se o modelo:

$$
\widehat{L D 4_{i}}=a+b_{1} I d_{i}+b_{2} P d_{i}+b_{3} \ln \left(T_{i}\right)+b_{4} I d_{i} \ln \left(T_{i}\right)
$$

onde os coeficientes $\mathbf{a}, \mathbf{b}_{1}, \mathbf{b}_{2}, \mathbf{b}_{3}$, e $\mathbf{b}_{4}$ estimam os parâmetros a, $\mathbf{B}_{1}, \mathbf{B}_{2}, \mathbf{B}_{3}, \mathrm{e} \mathbf{B}_{4}$.

O programa estatístico utilizado para os cálculos do modelo de regressão foi o "SAS/stat, proc reg”, versão 6.03. O programa gráfico utilizado para as Figuras 1 e 2 foi o "SAS/graph, proc gplot", versão 6.03. A Figura 7 foi feita a partir do programa gráfico "SAS/graph, proc g3grid, proc g3d", versão 6.03. As figuras 3, 4, 5 e 6 foram elaboradas com o programa gráfico " $S A S / g r a p h$, proc gchart", versão 6.03. Utilizou-se nos cálculos e na produção das figuras um microcomputador IBM 386 DX.

\section{RESULTADOS}

A proporção de motoristas e cobradores na população estudada foi de 0,53 e 0,47 , respectivamente (147 motoristas e 131 cobradores).

As Figuras 3, 4, 5 e 6 mostram a distribuição das variáveis I, T, Pd e LD4, acima descritas, na população estudada.

A Tabela 2 resume os resultados obtidos na aplicação do modelo de regressão linear múltipla acima ajustado.

TABELA 2. Estimativas e Estatísticas Obtidas com a Aplicação do Modelo de Regressão

$$
\widehat{L D 4}{ }_{i}=a+b_{1} I d_{i}+b_{2} P d_{i}+b_{3} \ln \left(T_{i}\right)+b_{4} I d_{i} \ln \left(T_{i}\right)
$$

\begin{tabular}{llccc}
\hline \hline Parâmetro & Estimativa & Erro Padrão & $\begin{array}{c}\text { Valor de " } \mathrm{t} \text { " } \mathrm{p} / \\
\mathrm{H}: \text { Parâmetro }=0\end{array}$ & $\mathrm{p}=$ value \\
\hline$\alpha$ & $-9,49 \mathrm{~dB}$ & 9,5651 & $-0,992$ & 0,3221 \\
$\beta_{1}$ & $1,29 \mathrm{~dB} /$ ano & 0,2292 & 5,624 & 0,0001 \\
$\beta_{2}$ & $-0,17 \mathrm{~dB} / \mathrm{mmHg}$ & 0,0554 & $-3,049$ & 0,0025 \\
$\beta_{3}$ & $5,78 \mathrm{~dB} / \mathrm{ln}$ (mês) & 2,5146 & 2,300 & 0,0222 \\
$\beta_{4}$ & $-0,12 \mathrm{~dB} /$ ano,mês & 0,0621 & $-1,967$ & 0,0502 \\
\hline \hline
\end{tabular}

$$
\mathrm{r}^{2}=0,274 \quad \mathrm{~F}=25,739 ; \quad \text { nível de significância }<0,001
$$

Considerando que:

a) a análise de variância associada ao modelo representado pela equação (2) mostrou uma estatística $\mathrm{F}$ suficientemente grande $(25,739)$ para implicar num nível de significância menor que 0,001 ;

b) a análise dos resíduos mostrou que estes têm distribuição próxima da distribuição normal, além de dispersão razoavelmente uniforme quando plotados contra Id e $\ln (\mathrm{T})$;

c) são válidos os pressupostos do modelo de regressão múltipla.
Que pressupõe:

a) LD4 é variável aleatória observável;

b) Pd, Id e T são variáveis não-aleatórias;

c) e é variável aleatória não-observável;

d) $\alpha, \beta_{1}, \beta_{2}, \beta_{3}$ e $\beta_{4}$ são parâmetros desconheci$\operatorname{dos}\left(\alpha, \beta_{1}, \beta_{2}, \beta_{3}\right.$ e $\left.\beta_{4} \in \mathrm{R}\right)$;

e) $\mathrm{V}(\varepsilon)=$ constante, desconhecida;

f) $\varepsilon_{1}, \varepsilon_{2}, \ldots, \varepsilon_{278}$ são estatisticamente independentes;

g) $\mathrm{E}(\varepsilon)=\mathrm{E}\left(\varepsilon_{2}\right)=\ldots=\mathrm{E}\left(\varepsilon_{278}\right)=0$. 
Levando-se em consideração as estimativas apresentadas na Tabela 2, sob o modelo de re- gressão utilizado, pode-se afirmar, para a população estudada, que os dados suportam a expressão:

$$
\widehat{L D 4}=1,29 I d_{i}-0,17 P d_{i}+5,78 \ln \left(T_{i}\right)-0,12 I d_{i} \ln \left(T_{i}\right)
$$

(LD4 em dB, Id em anos, Pdem mmHg, T em meses)

FIGURA 3. Distribuição da Idade na População Estudada

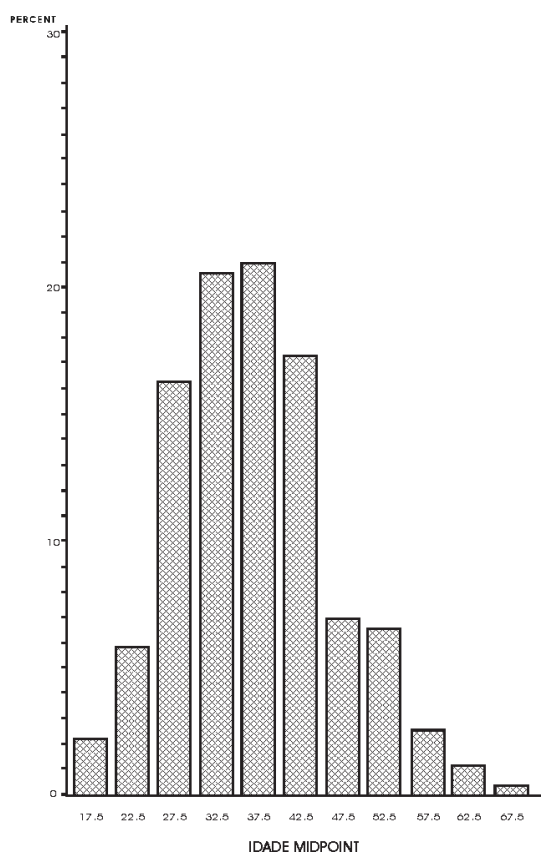

FIGURA 4. Distribuição do Tempo Total de Trabalho Acumulado como Condutor

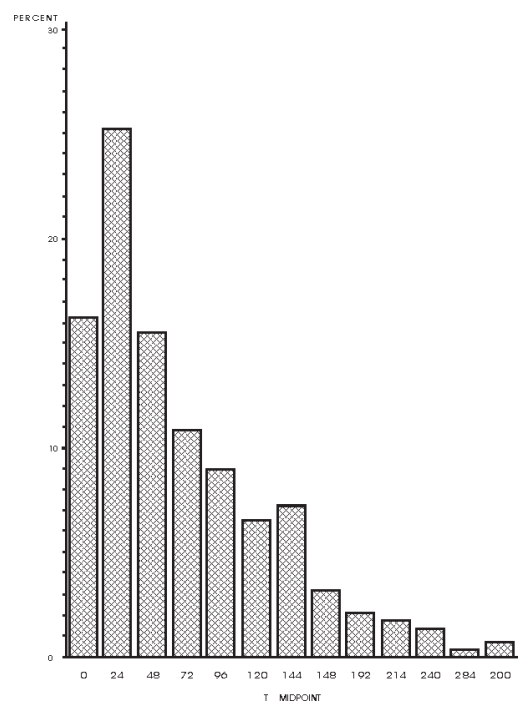


FIGURA 5. Distribuição da Pressão Arterial Diastólica na População Estudada

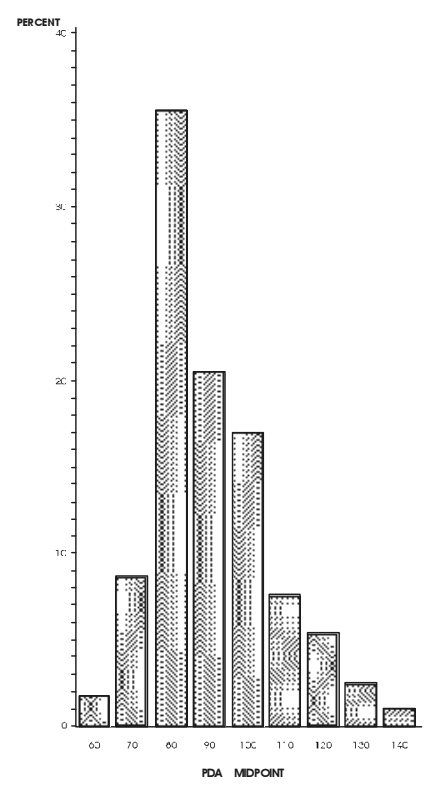

Pressão arterial diastólica em $\mathrm{mmHg}$

FIGURA 6. Distribuição do Limiar Auditivo na População Estudada - Ouvido Direito Freqüência de $4000 \mathrm{~Hz}$

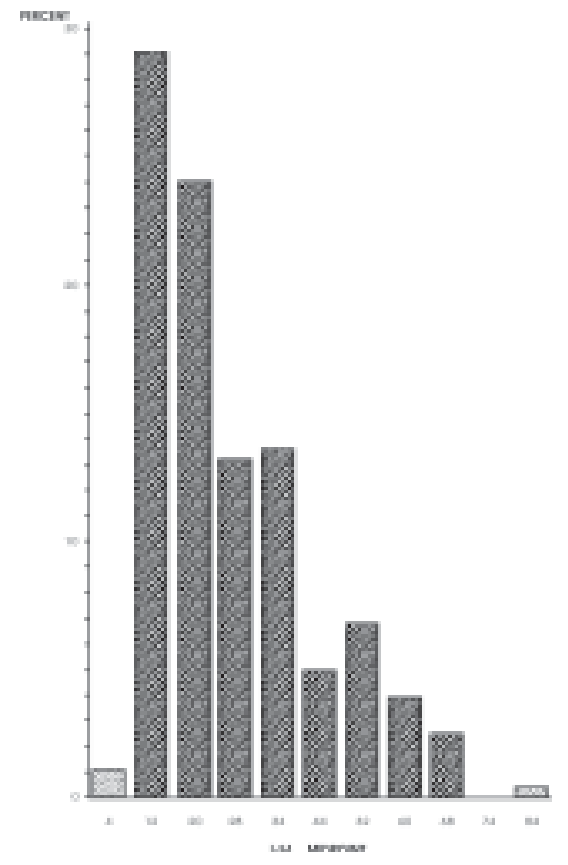

Pressão arterial diastólica em $\mathrm{mmHg}$ 


\section{DISCUSSÃO}

A equação (3) evidencia uma associação negativa entre o limiar auditivo no ouvido direito a $4000 \mathrm{~Hz}$ e a pressão arterial diastólica, para os trabalhadores da população estudada. Segundo ela, mantendo-se constantes as variáveis Id e $\mathrm{T}$, cada aumento de $1 \mathrm{mmHg}$ na pressão diastólica está associado a uma diminuição de 0,17 dB da variável LD4.

Tal achado não é referendado pela literatura especializada. Os estudos relacionando exposição ao ruído e pressão arterial são ainda controversos - embora já se possa vislumbrar uma tendência à identificação de associação positiva entre exposição profissional a ruído e prevalência de hipertensão arterial sistêmica (HAS) (Kristensen, 1989; Cordeiro et al., 1993). Estando já bem estabelecido um aumento da pressão sistólica como efeito da exposição aguda a alto nível de pressão sonora (Andren et al., 1982), há trabalhos que encontram associação significativa entre exposição crônica ao ruído e HAS (Parvizpoor, 1976; Jonsson \& Hansson, 1977; Manninen \& Aro, 1979), enquanto outros não conseguem identificar esta associação em níveis significativos (Shapiro, 1979). Fouriaud et al. (1984) encontraram associação significativa entre exposição profissional a ruído e prevalência de HAS entre trabalhadores de pequenas e médias empresas de Paris. Talbott et al. (1985) encontraram, entre trabalhadores de uma metalúrgica portadores de perda auditiva induzida pelo ruído em grau elevado, um aumento significativo da prevalência de HAS quando comparados com trabalhadores da mesma fábrica com audição normal. Wu et al. (1987), em criterioso estudo observacional realizado com trabalhadores de um estaleiro, utilizando modelagens distintas, concluiu que entre os expostos cronicamente a altos níveis de ruído, o risco de desenvolver HAS é cerca de duas vezes maior em relação aos não-expostos.

A equação (3) evidencia também uma associação positiva entre o limiar auditivo no ouvido direito a $4000 \mathrm{~Hz}$ e a idade, confirmando desta vez uma expectativa já bem estabelecida na literatura. De fato, segundo tal equação, mantendo-se constantes as variáveis $\mathrm{Pd}$ e $\mathrm{T}$, LD4 aumenta na medida em que aumenta Id. No entanto, o que chama a atenção é que este acréscimo de LD4 em função de Id não é constante, mas sua grandeza depende da magnitude do tempo acumulado envolvido. Tal comportamento é o reflexo da interação existente entre as variáveis Id e T, expressa na equação (3) pelo termo:

$$
-0,12 \operatorname{Id} d_{i} \ln \left(T_{i}\right)
$$

Assim, segundo o modelo ajustado, mantendo-se constante a $\mathrm{Pd}$, o acréscimo do limiar auditivo direito a $4000 \mathrm{~Hz}$, associado a um acréscimo de idade, é maior para os trabalhadores mais jovens na profissão do que para aqueles com maior experiência profissional.

A partir da equação (3), pode-se deixar o acréscimo no limiar auditivo (LD4) entre os instante 2 e 1 , mantendo-se a pressão diastólica constante, como:

$$
\Delta L D 4=1,29\left(I d_{2}-I d_{1}\right)+5,78 \ln \left[\frac{T_{2}}{T_{1}}\right]-0,12 \ln \left[\begin{array}{l}
\frac{T_{2}^{I d_{2}}}{T_{1}^{I d_{1}}}
\end{array}\right]
$$

onde $\operatorname{Id}_{1}$ e $\mathrm{Id}_{2}$ são as idades nos instantes le 2, e $\mathrm{T}_{1}$ e $\mathrm{T}_{2}$ são os tempos totais acumulados de trabalhos nos instantes 1 e 2 , respectivamente.

Analisando-se a expressão acima, percebese que, mantendo-se constante a diferença entre os valores $\mathrm{T}_{2}$ e $\mathrm{T}_{1}$, diferença esta que baliza o envelhecimento profissional do condutor, $o$ acréscimo de LD4 associado ao acréscimo de Id, envelhecimento biológico, diminui quan- do as faixas de tempo acumulado de trabalho aumentam.

Exemplificando, um trabalhador que aos 30 anos tem T igual a 24 meses, após trabalhar dez anos como condutor passa a ter T igual a 144 meses e Id igual a 40 anos. Segundo a equa- 
ção(5), o acréscimo predito em seu LD4 é de $10,84 \mathrm{~dB}$. No entanto, se este trabalhador tivesse aos 30 anos T igual a 120 meses (e não 24 meses como no caso anterior), após trabalhar os mesmos 10 anos teria também Id igual a 40 anos mas T igual a 240 meses. Neste caso, o acréscimo na variável LD4 seria de 7,83 dB. Percebe-se que o acréscimo do LD4 predito para o trabalhador em início de carreira excedeu em $38 \%$ aquele predito para o trabalhador mais velho na profissão (idade profissional), muito embora o tempo transcorrido entre $\mathrm{T}_{1} \mathrm{e}$ $\mathrm{T}_{2}$ e as idades tenham sido exatamente os mesmos nos dois casos.

Analogamente, na amplitude dos dados aqui trabalhados, considerando-se constante e variável Pd, observa-se, com base na equação (3), uma associação positiva entre a variável dependente LD4 e a variável preditora T. Isto é, observa-se que, independentemente da variável idade, LD4 aumenta na medida em que aumenta o tempo acumulado de trabalho. Não se encontra referência a tal associação na literatura especializada. Semelhantemente ao que ocorre na associação entre LD4 e Id, também a associação entre LD4 e T não é linear, mas sim modulada pela interação existente entre as variáveis $\mathrm{T}$ e Id. Esta interação faz com que a magnitude da variação do LD4, em função da vairável $T$, dependa também da faixa em que varia a idade do condutor.

Assim, segundo o modelo ajustado, mantendo-se constante a variável $\mathrm{Pd}$, o acréscimo do limiar auditivo a $4000 \mathrm{~Hz}$ no ouvido direito associado a um acréscimo constante da variável $\mathbf{T}$ é maior para os trabalhadores mais jovens do que para aqueles mais velhos.

Exemplificando, um trabalhador que aos 20 anos tem T igual a 24 meses, após trabalhar dez anos nesta profissão passa a ter T igual a 144 meses e Id igual a 30 anos. Segundo a equação (5), seu acréscimo predito para LD4 é de 12,99 dB. No entanto, se este trabalhador tivesse $T$ igual a 24 meses aos 40 anos (e não aos 20 como no caso anterior), após trabalhar os mesmos 10 anos teria também T igual a 144 meses mas Id igual a 50 anos. Neste caso, o acréscimo na variável LD4 seria de 9,79 dB. Percebe-se que o acréscimo do LD4 predito para o trabalhador mais jovem foi $33 \%$ maior que aquele predito para o mais velho, muito embora o tempo trabalhado e o acréscimo na idade tenham sido exatamente os mesmos nos dois casos.

A Figura 7 ilustra o que foi discutido acima. Ela reproduz a variação do valor predito para LD4 em função das variáveis Id e T, segundo a equação (5), mantendo-se constante a variável Pd.

Observa-se nesta figura que, na medida em que Id vai aumentando, os aumentos de LD4 associados a aumentos de T vão diminuindo, isto é, em idades menores a variação de LD4 em função de $\mathrm{T}$ é maior do que a ocorrida em idades maiores. Visto sob outra óptica, observa-se que o aumento de LD4 associado ao aumento de Id diminui na medida em que aumenta $\mathrm{T}$.

Outra observação a ser feita a partir desta figura é que, mantendo-se Id constante, o aumento instantâneo de LD4 associado a um aumento instantâneo de LD4 associado a um aumento de $\mathrm{T}$ vai diminuindo na medida em que a própria variável T aumenta, reflexo do "efeito logarítmico" desta variável sobre LD4.

As associações acima descritas mantêm-se qualitativamente as mesmas quando se analisam em separado motoristas e cobradores, 0 que sugere que seus determinantes devam ser comuns aos dois grupos.

Diferentemente dos ensaios clínicos, onde a alocação da população estudada é aleatória (o que cria instrumentos para a mensuração da variabilidade associada ao acaso, além de impedir eventual tendência na seleção), este é um estudo onde os trabalhadores da população estudada não foram randomicamente alocados. Tratando-se de um estudo transversal com população não-aleatorizada (usuários de um serviço de saúde), a interpretação dos dados aqui apresentados deve ser precedida da verificação cuidadosa da existência de potenciais vieses de seleção, o que em nosso entendimento não é tarefa fácil. Acreditamos que não houve tendenciosidade na seleção de casos, uma vez que foram alocados todos os motoristas e condutores que procuraram o PST, que por sua vez indicava a realização de exame audiométrico também em todos os condutores. Tais exames não foram realizados apenas em circunstâncias fortuitas, tais 
como períodos de manutenção técnica do audiômetro ou falta do funcionário especi- alizado para a realização da audiometria, o que não implicou erro sistemático de seleção.

FIGURA 7. Limiar Auditivo no Ouvido Direito X Idade X Tempo Acumulado de Trabalho na População Estudada

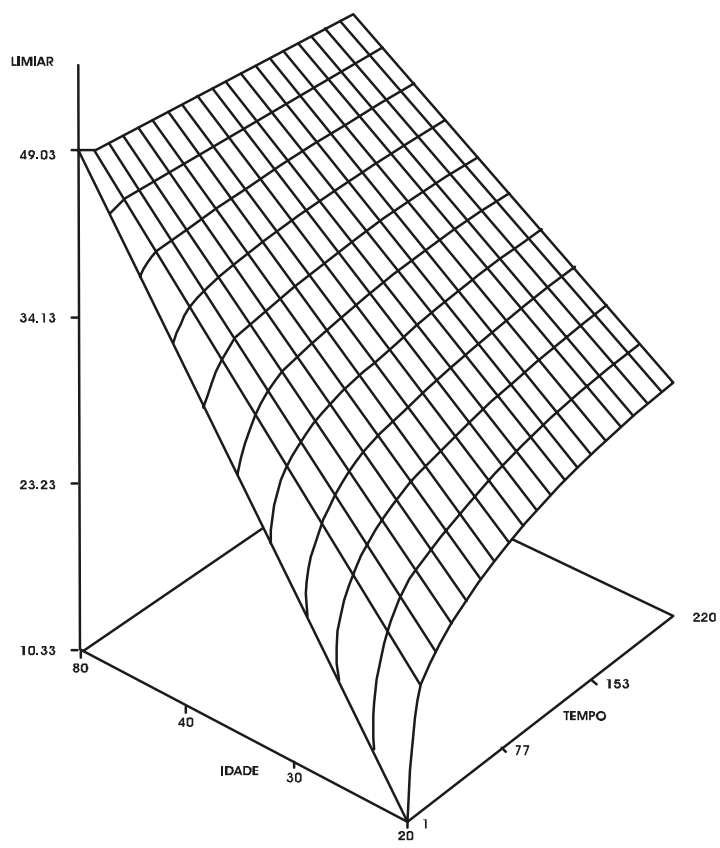

Limiar Auditivo - em dB, medido a $4000 \mathrm{~Hz}$

Tempo Acumulado - em meses

Em 1991, Correa-Filho et al. realizaram estudo transversal na cidade de Campinas, com população randomicamente alocada, onde a prevalência encontrada de perda auditiva induzida pelo ruído, bem como de hipertensão arterial sistêmica, foi bastante próxima da encontrada aqui.

Após estas considerações, na interpretação dos achados apresentados, algumas hipóteses podem ser levantadas. A primeira delas seria que eles refletem o efeito da "evasão" da força de trabalho daqueles trabalhadores que apresentam alterações audiométricas (que se sabe ocorrem acompanhadas de outras alterações de saúde) na medida em que foram exercendo sua profissão. Tal fenômeno, conhecido como "efeito do trabalhador sadio" (McMichael et al., 1975), se dá quer por incapacidade própria do trabalhador em manter-se em atividade, quer
Idade - em anos

Pressão Arterial Diastólica $=90 \mathrm{mmHg}$

por interferência direta dos serviços de saúde das empresas, que selecionam ativamente os mais "hígidos" para o trabalho.

Outra hipótese se prende à própria história natural da perda auditiva induzida pelo ruído, que tem um curso mais intenso nos primeiros anos de sua instalação, apresentando evolução mais insidiosa com o decorrer do tempo (Melnick, 1978).

Outra hipótese, ainda, seria a melhoria das condições de trabalho, particularmente as relacionadas à diminuição da jornada de trabalho e à diminuição do ruído produzido pelos ônibus, que vêm ocorrendo nos últimos anos na cidade de Campinas, o que tornaria o exercício da profissão menos "patogênico" em anos mais recentes.

Por fim, deve-se salientar o achado mais importante deste estudo não são as associações 
entre o limiar auditivo e as variáveis preditoras, mas antes disso, a própria existência de um significativo número de trabalhadores com perdas auditivas seguramente decorrentes de exposição profissional a ruído excessivo, fato este que nos remete obrigatoriamente ao campo das ações no sentido de bloquear esta agressão. E, neste sentido, a própria categoria dos condutores já vem apontando o que, segundo ela, seria a solução do problema: renovação da frota e diminuição da jornada de trabalho.

\section{AGRADECIMENTOS}

Os autores agradecem ao Programa de Saúde do Trabalhador de Campinas pela cessão dos dados clínicos e audiométricos para a realização deste estudo, e ao Laboratório de Aplicação em Epidemiologia (Lape), do DMPS/FCM/ Unicamp, pelo apoio computacional na análise dos dados aqui apresentados.

\section{RESUMO}

CORDEIRO, R.; LIMA-FILHO, E.C. \& NASCIMENTO, L. C. R. Associação da Perda Auditiva Induzida pelo Ruído como Tempo Acumulado de Trabalho entre Motoristas e Cobradores. Cad. Saúde Públ., Rio de Janeiro, 10 (2): 210-221, abr/jun, 1994.

Através da técnica da regressão linear múltipla, estudou-se a relação existente entre a perda auditiva induzida pelo ruído e as variáveis "tempo total acumulado de trabalho como condutor de veículos coletivos urbanos", "pressão arterial diastólica" e "idade", em uma população de 278 motoristas e cobradores usuários de um serviço de saúde ocupacional da cidade de Campinas, estado de São Paulo (Brasil).

Os principais resultados encontrados foram uma associação positiva entre a perda auditiva e o tempo acumulado de trabalho, bem como a existência de uma interação entre esta variável e a idade dos condutores.

Palavras-Chave: Epidemiologia; Regressão Linear; Ruído, Perda Auditiva; Saúde do Trabalhador

\section{REFERÊNCIAS BIBLIOGRÁFICAS}

ANDREN, N.; LINDSTEDT, G.; BJÖRKMAN, M.; BORG, K. O. \& HANSSON, L., 1982. Effecto of noise on blood pressure and "stress" hormones. Clinical Science, 62: 137-141.

ASTETE, M. G. W. \& KITAMURA, S.,1980. Efeitos da exposição ao barulho. In: Medicina do Trabalho. Doenças Profissionais (R. Mendes, ed.), pp. 415-436, São Paulo: Sarvier.

ATTIAS, J. \& PRATT, H., 1985. Auditoryevoked potential correlates of susceptibility to noise-induced hearing loss. Audiology, 24: 149-156.

BARONE, J. A.; PETERS, J. M.; GARABRANT, D. H.; BERNSTEIN, L. \& KREBSBACH, R., 1987. Smoking as arisk factor in noise-induced Hearing loss. Fournal of Occupational Medicine, 29: 741-745.

BROWNING, G. G.; GATEHOUSE, S. \& LOWE, G. D. O., 1986. Blood viscosity as a factor in sensorimental hearing impairment. The Lancet, 18: 121-123.

CARTER, N. L., 1980. Eye colour and susceptibility to noise-induced permanent threshold shift.Audiology, 19: 86-93.

CESANA, G. C.; FERRARIO, M.; CURTI, R.; ZANETTINI, R.; GRIECO, A.; PALERMO, A.; MARA, G.; LIBRETTI, A. \& ALGERI, S., 1982. Lavoro e stress. Escrezione urinaria di catecolamine in operai turnisti esposti a rumore. I: Adrenalina e noradrenalina. Medicina del Lavoro, 73: 99-109.

CESANA, G. C.; PANZA, G.; FERRARIO, M.; GRIECO, A.; SEGA, R.; FOLCINI, V.; DAMIANI, e. \& LIBRETTI, A., 1983. Attività MAO piastrinica in lavoratori turnisti esposti a rumore. Medicina del Lavoro, 74: 266-271.

CORDEIRO, R., 1991. Pressão Arterial Diastólica entre Motoristas e Cobradores de Campinas, Usuários de um Serviço de Saúde Ocupacional. Tese de Mestrado, Campinas: Faculdade de Ciências Médicas da Universidade Estadual de Campinas. 
CORDEIRO, R., FISCHER, F. M., LIMAFILHO, E. C. \& MOREIRA-FILHO, D. C., 1993. Ocupação e hipertensão. Revista de Saúde Pública, 27: 380-387.

CORREA-FILHO, H. R.; NASCIMENTO, L. C. R.; SALERNO, V. L. \& RIMOLI, J., 1991. Surdez Ocupacional e Hipertensão Arterial em Condutores de Ônibus Urbanos em Campinas, São Paulo, Brasil. Trabalho apresentado no IV Congresso Paulista de Saúde Pública. São Paulo: Associação Paulista de Saúde Pública, 10 e 14 de julho. (Mimeo).

FOURIAUD, C.; JACQUINET-SALORD, $M$. C.; DEGOULET, P.; AIME, F.; LANG, T.; LAPRUGNE, J.; MAIN, J.; OECONOMOS, J.; PHALENTE, J. \& PRADES, A.; 1984. Influence of socioprofessional conditions on blood pressure levels and hipertension control. American Fournal of Epidemiology, 120: 72-86.

FRIEDMAN, S. A.; SCHULMAN, R. H. \& WEISS, S., 1975. Hearing and diabetie neuropathy. Archives of Internal Medicine, 135: 573-576.

HARNER, S. G., 1981. Hearing in adult - onset diabetes mellitus. Otolaryngology on Head and Neck Surgery, 89: 322-327.

HODGSON, M. J.; TALBOTT, E.; HELMKAMP, J. C. \& KULLER, L. H., 1987. Diabetes, noise exposure, and hearing loss. Fournal of Occupational Medicine, 29: 576-579.

HUNGRIA, H., 1987. Otorrinolaringologia. $5^{\text {a }}$ ed., Rio de Janeiro: Guanabara.

JONSSON, A. \& HANSSON, L., 1977. Prolongued exposure to a stressfull stimulus (noise) as a cause of raised blood pressure in man. The Lancet, 1: 86-87.

KRISTENSEN, T. S., 1989. Cardiovascular diseases and the work enviromment - a critical review of the epidemiologie literature on monchemical factors. Scandinavian Fournal of Work and Envirommental Health, 15: 165-179.

LAURELL, A. C. \& NORIEGA, M., 1987. Trabajo y Salud en Sicartsa. México: Programa de Difusión Cultural del SITUAM.
MANNINEN, O. \& ARO, S., 1979. Noiseinduced hearing loss and blood pressure. International Archives of Occupational Envirommental Health, 42: 251-256.

McMICHAEL, A. J.; HAYNES, S. G. \& TYROLER, H. A., 1975. Observations on the evalution of occupational mortality data. Fournal of Occupational Medicine, 17: 128-131.

MELNICK, W., 1978. Temporary and pemanent threshold shift. In: Noise and Audiology (D. M. Lipscomb, ed.), pp. 115125, Baltimore: University Park Press.

MERLUZZI, F; CORNACCHIA, L.; PARIGI, G. \& TERRANA, T., 1979. Metodologia di esecuzione del controllo dell' udito dei lavoratori esposti a rumore. Nuovo Archivio Italiano di Otologia, 7: 695-714.

PARVIZPOOR, D., 1976. Noise-induced hearing loss and blood pressure. International Archivesof Occupational and Envirommental Health, 42: 251-256.

RAI, R. M.; SINGH, A. P.; UPADHYAY, T. N.; PATIL, S. K. B. \& NAYAR, H. S., 1981. Biochemical effects of chronic exposure to noise in man. International Archives of Environmental Health, 48: 331-337.

SHAPIRO, A. P., 1979. The role of the stress in hypertension. Fournal of Human Stress, 5:7-26.

SIEGELAUB, A. B.; FRIEDMAN, G. D.; ADOUR, K.; CALIF, O. \& SELTZER, C. C., 1974. Hearing loss in adults - relation to age, sex, exposure to loud noise and cigarette smoking. Archives of Environmental Health, 29: 107-114.

TALBOTT, E.; HELMKAMP, J.; MATTHEWS, K.; KULLER, L.; COTTINGTON, E. \& REDMOND, G., 1985. Occupational noise exposure, noiseinduced hearing loss, and the epidemiology of high blood pressure. American fournal of Epidemiology, 121: 501-514.

WU, T.; KO, Y. \& CHANG, P., 1987. Study of noise exposure and high blood pressure in shipyard workers. American fournal of Industrial Medicine, 12: 431-438. 